\title{
Optical H2 sensing properties of vertically aligned Pd/WO3 nanorods thin films deposited via glancing angle rf magnetron sputtering
}

\begin{abstract}
In this work, the optical $\mathrm{H} 2$-sensing properties of $\mathrm{Pd} /$ tungsten trioxide (WO3) nanorods prepared by rf magnetron sputtering with glancing-angle deposition (GLAD) technique are investigated. From grazing-incidence $\mathrm{X}$-ray diffraction and field emission scanning electron microscopic characterizations, annealed WO3 structure deposited on a quartz substrate at glancing angle of $85^{\circ}$ exhibited polycrystalline monoclinic crystal structure with uniform partially isolated columnar nanorod morphology. The nanorods have the average length, diameter and rod separation of around $400 \mathrm{~nm}, 50 \mathrm{~nm}$ and $20 \mathrm{~nm}$, respectively. The developed sensors show remarkable gasochromic absorbance response when exposed to $\mathrm{H} 2$. Cumulative absorbance in 650-1000 nm wavelength range is increased by approximately $51 \%$ toward $\mathrm{H} 2$ with $0.1 \%$ concentration in synthetic air, which is more than an order of magnitude higher than that of WO3 dense film prepared by conventional sputtering method. Moreover, WO3 nanorod based sensor is much more promising for practical use due to its much faster response. Therefore, the developed Pd/WO3 nanorod based optical sensors are highly potential for low $\mathrm{H} 2$ concentration sensing with highly sensitivity, fast and stable responses and low operating temperature.
\end{abstract}

Keyword: Tungsten trioxide; Glancing-angle deposition; Nanorods; H2 sensor; Optical gas sensor 